\title{
Fin de l'histoire et nouveaux temps : le PCF et l'intégration européenne du traité de Maastricht au rejet du traité constitutionnel européen (1992-2005)
}

\section{End of the Past and a New Era. The French Communist Party and European Integration: from the Maastricht Treaty to the rejection of the European Constitutional Treaty (1992-2005)}

\begin{abstract}
Émilia Robin
UMR Sorbonne Identités, relations internationales et civilisations de l'Europe,

Francia

Emilia.robin@free.fr https://orcid.org/0000-0003-4327-2260
\end{abstract}

Recibido: 16/07/2021

Aceptado: 15/12/2021

Cómo citar este artículo: ROBIN, Émilia (2022). Fin de l'histoire et nouveaux temps : le PCF et l'intégration européenne du traité de Maastricht au rejet du traité constitutionnel européen (1992-2005). Pasado y Memoria. Revista de Historia Contemporánea, (24), pp. 42-65, https://doi.org/1014198/PASADO2022.24.02

Résumé

En 1992, comme en 2005, le Parti Communiste Français (PCF) appelle à voter contre les traités européens soumis à référendum (Maastrich et Constitution européenne) dans la continuité de son rejet traditionnel de l'intégration européenne. Cependant, la nature de ce rejet évolue considérablement au cours de la période. D’une posture quasi souverainiste et qualifiée d'« eurosceptique »dans les années 1990, il passe à une analyse articulée autour de l'anti-libéralisme. Cette évolution est à la fois la cause 
et la conséquence de la mutation interne du PCF après la guerre froide, des recompositions idéologiques de la gauche, et de l'européanisation de la vie politique nationale.

Mots-clés: France; Union Européenne; Communisme; Anti-liberalisme.

\title{
Resumen
}

En 1992 y en 2005, el PCF llamó a votar contra los tratados europeos sometidos a referéndum en su tradicional rechazo a la integración europea. Sin embargo, la naturaleza de esta oposición cambió considerablemente a lo largo del período. De una postura casi soberanista y calificada de «euroescéptica» en la década de los noventa, pasó a un análisis basado en el antiliberalismo. Esta evolución fue tanto la causa como la consecuencia de la transformación interna del PCF después de la Guerra Fría, de las recomposiciones ideológicas de la izquierda y de la europeización de la vida política nacional.

Palabras clave: Francia; Unión Europea; Comunismo; Anti-liberalismo.

\begin{abstract}
In 1992 and in 2005, the Parti Communiste Francais (PCF) urged to vote against the European treaties then submitted to referendum (Maastricht and European Constitution) in line with the Party's traditional rejection of European integration. However, the nature of said rejection gradually underwent a major evolution along that period. From a rather sovereignist stance, qualified as «Eurosceptic», along the 1990s, it moved on to an analysis articulated around the concept of anti-liberalism. Such development was both the cause and the consequence of the PCF internal transformation after the Cold War, the ideological reconfigurations of the left and, finally, the Europeanization of national political life.
\end{abstract}

Keywords: France; European Union; Communism; Anti-Liberalism.

Le 20 septembre 1992, le traité de Maastricht est adopté par référendum avec une courte avance ( $51 \%$ des voix), à la suite d'un débat intense où le PCF défend des positions souverainistes que beaucoup de commentateurs associent aux thèses d'une partie de la droite. Le 29 mai 2005, le traité établissant une Constitution pour l'Europe est rejeté par 54,7\% des votants, le PCF s'associant à un vaste mouvement de gauche fédéré par l'antilibéralisme. Entre-temps, le parti s'est profondément transformé, en même temps que son influence reculait.

Deux histoires parallèles et contraires se développent donc entre 1992 et 2005, ponctuées de consultations électorales à divers échelons, des municipales aux européennes en passant par les cantonales, les législatives et les présidentielles. Ces consultations successives imposent leur rythme et 
remplissent plusieurs fonctions pour le PCF : elles permettent de donner une visibilité nationale à son programme politique, et de mesurer son audience et son influence. Les élections sont également un moment de mobilisation des militants et un espace de débat permettant des formes d'ouverture et d'alliances stratégiques, notamment sur le terrain. Et de ce point de vue, il s'avère que les rendez-vous européens sont des moments-clés dans l'histoire du PCF : c'est cette articulation paradoxale que nous allons tenter d'expliquer.

Il est en effet paradoxal que l'Europe joue un rôle structurant dans l'évolution du PCF, alors que son programme politique était - et reste - hostile à la construction européenne telle qu'elle se fait. Par conséquent, comment comprendre que le rapport du PCF à l'UE ait évolué ? Pour mieux le comprendre, nous nous appuierons sur une source originale et peu étudiée, le mensuel de l'Association nationale des élus communistes et républicains (ANECR), créé en 1978 et disparu en 2018. Ce magazine peu connu avait une fonction utilitaire plus que polémique, et servait de bulletin d'information aux adhérents de l'ANECR, principalement des élus municipaux. Officiellement, ils étaient 22000 élus en $1989^{1}$, et en 2021 le PCF en revendique 7500². L'Élu d'aujourd'hui propose des articles argumentatifs sur la construction européenne, tout en cherchant à étoffer et enrichir l'argumentaire des militants. C'est donc une source précieuse pour mesurer le rôle du thème européen dans les réflexions du PCF.

Nous nous pencherons successivement sur l'évolution du PCF en tant que parti et que force politique ; l'évolution de sa perception de l'UE, grâce notamment au développement d'une expertise interne ; la question de l'euroscepticisme ; et enfin, le rôle stratégique du thème européen pour un petit parti.

\section{Le PCF entre déclin et mutation}

Dès le début des années 1980, l'audience du PCF commence à diminuer 3 . Ce déclin se mesure au fil des consultations électorales, et frappe par sa rapidité : 4,4 millions de voix pour le PCF aux élections européennes de 1979, 2,2 millions seulement en 1984. À partir de 1989, le parti communiste passe sous le seuil des $10 \%$ de votants à toutes les consultations nationales et continue à

1. «L'enjeu européen, au quotidien », L'Élu d'aujourd'hui, no 126, mai 1989, p. 24.

2. «Les élus du PCF », https://www.pcf.fr/les_elus [consulté le 23 novembre 2021].

3. L'histoire du parti communiste a fait l'objet de nombreuses études et d'un profond renouvellement grâce aux apports de la sociologie historique. Le très récent ouvrage de Roger Martelli, Jean Vigreux et Serge Wolikow, Le Parti rouge. Une histoire du PCF, 1920-2020, propose la synthèse la plus à jour de ces travaux, et un bilan historiographique très complet. 
régresser dans les votes. Une légère embellie électorale se produit en 19971998, mais dans les années 2000, il frôle la catastrophe à deux reprises. Après la présidentielle de 2002, son trop faible score au premier tour $(3,4 \%$ des suffrages) lui interdit de bénéficier du remboursement des frais de campagne que la loi prévoit pour les partis dépassant $5 \%$ des suffrages. Et à l'issue des législatives de 2007, les 15 députés communistes ne sont pas assez nombreux pour permettre la formation d'un groupe parlementaire.

Plusieurs phénomènes interdépendants se conjuguent pour expliquer ce déclin : la longue fidélité du PCF à Moscou, le déclin du soutien ouvrier, la disparition des moyens d'action du PCF sur le terrain, les transformations de la société française, et ses choix d'alliances stratégiques.

Tout d'abord, le PCF est victime de sa lenteur à se distancier de l'URSS, alors qu'une série d'événements internationaux soulèvent l'indignation de l'opinion publique, notamment la répression du printemps de Prague en 1968, l'invasion de l'Afghanistan en 1979, la proclamation de l'état d'urgence en Pologne en 1981. Quelques années plus tard, la désillusion s'accentue avec l'effondrement des régimes communistes en Europe de l'Est. Lopinion publique découvre l'ampleur de leurs échecs économiques, sociaux et environnementaux, tandis que les travaux se multiplient sur leur nature dictatoriale, avec un ouvrage emblématique en France, Le livre noir du communisme, sorti en 1997. À cette date, le PCF a commencé une « mutation » portée par son nouveau secrétaire général, mais l'image d'un parti ancré dans le passé reste prégnante.

Deuxièmement, dès les années 1980, le PCF est confronté à l'effritement de ses bastions sociologiques causé par la désindustrialisation, notamment sidérurgique et minière. Non seulement la part des ouvriers dans la société française diminue, mais leur vote se reporte vers d'autres partis, notamment le Front national : en 1995, 27 \% des ouvriers votent pour le FN, ils sont $50 \%$ en 2019 (Martelli ; Vigreux; Wolikow, 2020 : 252). Le Front national récupère en réalité la fonction tribunitienne que le PCF a longtemps assumée, en devenant le réceptacle et le porte-parole d'un groupe social caractérisé par un sentiment de déclassement et d'abandon.

Cette évolution est à la fois la cause et la conséquence d'un troisième phénomène, «la dislocation du système d'action communiste » à partir de la fin des années 1970 (Mischi, 2007). Le processus s'accélère dans les années 1990, qui voient s'évanouir le modèle traditionnel d'acculturation et de formation politique du PCF : fermeture des écoles du parti, déstructuration du champ des penseurs affiliés, disparition du potentiel interne de production théorique avec la fin ou le sommeil des centres de recherche et des revues théoriques, disparition ou désaffiliation des organisations de masse - femmes, 
jeunes, mouvements de la paix, Secours populaire... - et des syndicats - à partir de 1993, la CGT n'appelle plus à voter communiste (Martelli ; Vigreux ; Wolikow, 2020 : 248). La permanence de L'élu d'aujourd'hui n'en est que plus frappante : créée en 1978, la revue paraît jusqu'en février 2018, avant d'être victime de ses difficultés financières ${ }^{4}$. LANECR, quant à elle, existe toujours en 2021, ainsi que son centre de formation, le CIDEFE (Centre d'information, de documentation, d'études et de formation des élus).

Une quatrième gamme d'explications porte sur l'adéquation du parti à la société française. Marie-Claire Lavabre et François Platone parlent à ce sujet d'« occasions manquées » (Lavabre; Platone, 2003). Sur de nombreux sujets en effet, le PCF privilégie l'approche idéologique qui donne la priorité à l'analyse de classes. C'est pourquoi il prend avec retard la mesure des évolutions de la société. Ce fut le cas en mai 1968 où il tarde à mesurer l'ampleur et la nature de la révolte étudiante, dans les années 1970 avec les luttes féministes, puis à partir des années 1980 autour des enjeux croisés liés à l'immigration (racisme, question des banlieues, diversité).

Cela ne signifie pas que les élus communistes soient aveugles aux évolutions de la société et à ses attentes. Lors du congrès de l'ANECR en 2001, Maxime Camuzat, maire de Saint-Germain-au-Puy et conseiller général du Cher, interrogeait en ces termes la lisibilité du discours du PCF : « comment faire passer notre choix stratégique d'être à la fois critiques et constructifs, contestataires de l'ordre établi et force de proposition ? $»^{5}$ Plus largement, il souligne la difficulté à créer un sentiment d'appartenance locale qui justifierait la participation à la vie politique, alors que les vies professionnelle et personnelle se déroulent dans des villes différentes, et que les intercommunalités dépossèdent les municipalités de leurs capacités d'action.

Mais de quel PCF parle-t-on ? Le déclin électoral et la perte d'influence du parti communiste, dans un contexte national et international bouleversé, sont la toile de fond d'une profonde transformation du parti à partir de janvier 1994, avec la désignation de Robert Hue comme secrétaire général, succédant à Georges Marchais qui occupait ce poste depuis décembre 1972.

De congrès en congrès, l'on fait évoluer à la fois la doctrine et l'organisation du parti. Les abandons doctrinaux avaient commencé dans les années 1960 : abandon de l'objectif du parti unique en 1963, renonciation à l'idée de dictature du prolétariat comme étape indispensable vers le socialisme en 1976, fin de la référence marxiste-léniniste en 1979. En 1994, le PCF abandonne le concept

4. «L'Élu d'aujourd'hui jette l'éponge », Presse News, no 555, 27 février 2018.

5. L'Élu d'aujourd'hui, no 260, juillet-août 2001, p. 38. 
de « rôle dirigeant de la classe ouvrière » et cesse de se définir comme "parti de la classe ouvrière » : cette décision est conforme à l'évolution de la société française et à la réalité de l'électorat communiste, mais elle rompt avec ce qui avait été le fondement identitaire du parti.

Du point de vue organisationnel, on abandonne en 1994 le centralisme démocratique, permettant désormais l'expression publique des divergences. Les nouveaux noms des instances dirigeantes (Bureau national, comité national, secrétaire national) symbolisent la rupture avec l'organisation ancienne. Le Comité national double ses effectifs et passe à 271 membres, dont beaucoup sont issus du « mouvement social » au sens large et ne sont pas nécessairement membres du PCF.

Enfin, le Congrès de Martigues en 2000 promeut « un vaste mouvement d'ouverture sur l'extérieur, donné comme principe de l'élaboration des nouveaux statuts » (Lavabre; Platone, 2003 : 35). Les militants ont une totale liberté d'organisation sur le terrain, la seule obligation étant d'informer le parti des décisions :

« Le changement proposé part d'un choix majeur : la primauté de l'adhérent. Il s'agit de fonder la conception de l'organisation non plus sur des structures mais sur les attentes, les motivations et la liberté d'engagement des femmes et des hommes communistes. À chacune et chacun de décider où, quand et comment s'impliquer, de choisir selon ses centres d'intérêt le cadre d'activité qui correspond à ses motivations : dans le milieu de travail, le voisinage, selon ses réseaux personnels d'activité, dans des formes qui correspondent à ses souhaits. Au Parti communiste de mettre en place sur tout le territoire national des lieux d'activité et de proximité. ${ }^{6}$

Bernard Pudal a résumé dans une formule frappante le résultat de cette évolution : vers 2007, le PCF est « une sorte de constellation de petites entreprises quasi individuelles ou collectives, à base locale ou idéologique, dont les liens sont plus ou moins forts avec les fédérations et la direction » (Pudal, 2009 : 177-178).

\section{Une nouvelle grille d'analyse de l'Union européenne}

Le parti communiste français a développé, au cours de son histoire, une perception à la fois idéologique et géopolitique de l'intégration européenne. Mais avec la fin de la guerre froide, cette perception évolue considérablement dans sa structure comme dans ses fondements. La mutation du PCF et le changement

6. Transparence, no 10, février-mars 2000, base commune de discussion en vue du congrès de Martigues, p. 16. 
de nature du projet européen contribuent à l'expliquer, mais il faut aussi tenir compte de l'appropriation directe du système politique de l'UE par le parti.

La perception communiste de la construction européenne s'est structurée à partir de la fin des années 1940, quand les premières institutions européennes voient le jour : Conseil de l'Europe, CECA, puis Marché commun. Dans un contexte de guerre froide, cette perception mêle étroitement préoccupations géopolitiques, héritages historiques et considérations idéologiques.

On peut considérer qu'elle s'articule autour de quatre thèmes (Robin, 2009). C'est tout d'abord la lutte des classes, grille de lecture fondamentale des rapports sociaux : un opuscule de Lénine, Du mot d'ordre des États-Unis d'Europe, paru en 1916, postule qu'un rapprochement entre États européens ne peut être qu'une « entente de capitalistes européens... dans le seul but d'étouffer en commun le socialisme en Europe ». L'on rencontre donc dès les années 1950 les expressions d'« Europe du patronat », « Europe des cartels », etc. Deuxième thème, trouvant également sa justification théorique dans le pamphlet de Lénine : l'anti-colonialisme. Pour le PCF, l'Europe est une union des anciennes puissances coloniales qui perpétuent à travers la CEE les rapports inégaux d'antan. Le troisième thème est celui de la défense de l'Union soviétique, qui signifie la condamnation de toute organisation ou structure internationale dont l'URSS ne serait pas partie prenante : l'Europe sans l'URSS est une Europe contre l'URSS. Enfin, quatrième thème, la construction européenne est le moyen pour la RFA non dénazifiée de poursuivre une politique de domination en Europe, désormais par le biais de l'économie. S'opposer à la construction européenne, c'est perpétuer la défense de la souveraineté nationale dans la continuité directe de la Résistance. En réalité, cette perception n'a rien de spécifique : appliqué au cas de la construction européenne, c'est tout simplement le programme communiste en relations internationales.

Avec la fin de la guerre froide, ce schéma est-il encore valide ? Certains mots d'ordre d'hier se rencontrent aujourd'hui, et les quatre thèmes d'hier n'ont pas disparu. La lutte des classes est à la fois transposée et amplifiée dans la lutte contre la mondialisation néo-libérale qu'incarnerait l'Union européenne et ses politiques d'ouverture à la concurrence. Lalter-mondialisme a pris le relais de l'anti-colonialisme, à la fois en affirmant l'égale dignité des peuples et en appelant à réorganiser et réguler les flux commerciaux et financiers. La défense de l'URSS est évidemment caduque, mais la condamnation d'une Europe «fermée » ressurgit régulièrement. Enfin, le PCF endosse toujours résolument le rôle de défenseur de l'indépendance nationale.

Cependant, cette continuité thématique est donc trompeuse car elle masque deux évolutions fondamentales. La première évolution est qu'à partir 
des années 1960, le PCF commence à admettre la réalité du Marché commun, son existence « objective », suivant en cela les « thèses » formulées par l'Institut de l'économie mondiale de Moscou en 1962. Le Programme commun de la gauche, qui l'associe au Parti socialiste entre 1972 et 1977, contribue également à intégrer la CEE dans ses horizons d'analyse. C'est très net lors de la campagne contre le traité de Maastricht : tout en étant résolument hostile à l'Europe dans sa forme comme dans son contenu, il ne demande ni sa dissolution, ni le retrait de la France. Au contraire, Robert Hue évoque à l'été 1992 « une construction européenne alternative » passant par des échanges et coopérations entre collectivités locales « en Europe et entre les pays d'Europe et ceux du tiers monde ${ }^{7}$. Dans le même numéro, le portrait de Sylviane Ainardi, député PCF au Parlement européen et conseillère régionale en Midi-Pyrénées, est placé sous le grand titre : « Je suis contre Maastricht parce que je suis pour l'Europe. $»^{8}$

La seconde évolution majeure est que pour le PCF des années 1990-2000, la construction européenne n'est plus un objet géopolitique, un enjeu de politique internationale. Du temps de la guerre froide, il fallait prendre en compte la manière dont l'URSS, en tant que grande puissance, analysait l'intégration européenne, et le PCF en est évidemment libéré. D’autre part, malgré le développement de la PESC/PESD, l'UE ne parle pas d'une seule voix, voire se divise sur certains sujets, comme on a pu le constater en 2002-2003 à propos de la guerre d'Irak.

Cette disparition de la géopolitique provient surtout de la nature technicienne de l'UE et des traités, de l'importance des enjeux réglementaires et législatifs, qui renvoient à une conception politique (par exemple, la question des services publics) mais pas à une conception géopolitique (ou alors, sur des sujets précis, comme l'adhésion de la Turquie). Cela se reflète dans l'organisation même du PCF, comme le relève Nicolas Azam : en 1994 le parti crée un « collectif de coordination pour les questions européennes ", qui devient « commission Europe » en 2000. En réalité, cette commission fonctionne avec un seul permanent et se réunit de manière irrégulière, mais sa simple existence prouve « la dissociation entre l'intégration européenne et le domaine de la politique étrangère... dans l'organigramme » (Azam, 2017 : 623).

Un second facteur contribue à faire évoluer la perception que le PCF a de l'intégration européenne : sa participation au fonctionnement des institutions à travers le Parlement européen, qui lui permet de construire progressivement une expertise propre sur l'Europe.

7. Éditorial de Robert Hue, «Le non des élus », L'Élu d'aujourd'hui, no 161, juillet -août 1992, p. 5.

8. Ibid., p. 13-15.

Pasado y Memoria. Revista de Historia Contemporánea, 24, 2022, pp. 42-65 
Le PCF participe activement à la vie parlementaire européenne, la figure emblématique étant Francis Wurtz, député européen pendant trente ans et président du GUE/NGL de 1999 à 2009. À travers ses représentants au Parlement européen, le parti développe progressivement une connaissance fine des rouages de l'UE, comme l'ont montré Willy Beauvallet et Sébastien Michon dans leur étude sur les députés européens du GUE/NGL (Beauvallet ; Michon, 2013).Dans les débats, la posture critique de ses élus est tout à fait identifiable. Mais ils jouent également le jeu du Parlement, avec un très grand sérieux dans la participation au travail parlementaire, supérieure à la moyenne des députés européens.

D'autre part, les députés européens du PCF endossent un rôle important de relais et d'intermédiaires : relais et transmission d'information vers leurs réseaux militants, porte-paroles des positions de leur mouvance dans l'espace du Parlement européen, mises en contact dans le cadre des processus législatifs, etc. Pour reprendre la distinction classique de Hirschmann, les députés communistes au Parlement européen privilégient donc explicitement la prise de parole (voice) à l'absentéisme (exit). Un portrait de Sylvie Mayer, députée européenne et membre du Comité central illustre bien cette fonction de protestation et d'information :

«Elle [Sylvie Mayer] est donc à l'Assemblée européenne le porte-parole de toutes les catégories sociales qui souffrent de la politique communautaire [...]. Et plus encore que le simple porte-parole, celle qui permet de prendre la parole, qui informe sur les mauvais coups, qui agit pour des solidarités de lutte. Car la façon d'agir du groupe communiste introduit la démocratie au sein du fonctionnement de la CEE. Il est vrai que les députés communistes sont les seuls à pouvoir promouvoir l'expression des intéressés au sein de l'appareil européen car ils sont les seuls au contact direct des habitants et de leurs luttes. $»^{9}$

Quelques années plus tard, Francis Wurtz revendique cette posture d'opposition, garantie d'un débat démocratique :

« Les débats au Parlement européen sont souvent polémiques en superficie mais trop consensuels sur le fond, comme si les questions traitées ne renvoyaient pas à des choix politiques fondamentaux relevant d'options très différentes et même parfois d'intérêts diamétralement opposés. Je pense qu'un essor beaucoup plus vigoureux de l'esprit critique et un débat ouvertement contradictoire - et, au besoin, iconoclaste - sur les enjeux essentiels de notre temps serait un facteur de démocratie. ${ }^{10}$ »

9. «L'enjeu européen, au quotidien », L'Élu d'aujourd'hui, no 126, mai 1989, p. 24.

10. Francis Wurtz, "Se ressourcer auprès des gens ", L'Élu d'aujourd'hui, no 177, janvier 1994 , p. 75. 
Les élus communistes savent que leurs positions ont peu de chances d'être adoptées, mais leur activité permet de poser des jalons juridiques au service des revendications. Le Parlement est aussi un lieu d'expérimentation pour une forme différente de débat, basée sur la recherche d'un consensus et la prise en compte des spécificités nationales. René Piquet l'explique à propos du fonctionnement interne du GUE et des contenus différents que les mots « nation » et « fédéralisme » peuvent avoir pour des Français et des Belges :

« Le respect de l'identité de chacun nous permet de parvenir fréquemment à des positions communes. Je pense qu'il ne faut pas se fier à son jugement, mais écouter l'autre pour savoir ce qu'il est et ce qu'il veut vraiment et en juger ensuite. $»^{11}$

Cette expérience directe des rouages européens prend une place plus importante dans L'Élu d'aujourd'hui à partir du milieu des années 1990. En effet, autant entre 1989 et 1994 il n'évoquait la construction européenne qu'à l'occasion des élections européennes et de la campagne de Maastricht ; autant par la suite le magazine s'attache à informer ses lecteurs sur l'actualité de l'échelon décisionnel européen. La date n'est pas anodine : l'entrée en vigueur du traité de Maastricht fin 1993 coincide avec la transformation lancée par Robert Hue début 1994. À partir de l'automne 1994, une nouvelle rubrique intitulée « Assemblées » propose chaque mois une synthèse des travaux récents de l'Assemblée nationale, du Sénat et du Parlement européen. Le tout premier article est une présentation de l'activité des sept députés communistes à Strasbourg :
« Nous sommes conduits à donner notre opinion, à formuler des choix sur la politique telle qu'elle se construit dans les institutions et également à interve- nir, à dire et faire le lien avec les forces sociales, ce que sont nos choix sur les grandes questions économiques, monétaires, sociales, agricoles, culturelles... [...] Nous structurons notre activité en lien avec les organisations syndi- cales, les mouvements et les associations, sous forme de rencontres diverses et d'auditions. Nous sommes disponibles pour répondre à leurs besoins, à ceux du parti, pour informer, mais aussi disponibles pour travailler avec les parlementaires nationaux. $»^{12}$

Par la suite, les eurodéputés communistes, René Piquet, Aline Pailler, Francis Wurtz, Philippe Herzog, Sylviane Ainardi, etc., signent tour à tour des brèves sur les dossiers européens (libéralisation des transports aériens, critères de

11. René Piquet, « «L'écho des luttes qui résonne », L'Élu d’aujourd'hui, no 169, avril 1993, p. 13.

12. Mireille Elmalan, « Avec le groupe confédéral de la Gauche unitaire européenne, de nouvelles possibilités d'action », L'Élu d'aujourd'hui, no 186, novembre 1994, p. 64. 
convergence...), des sujets internationaux (Algérie, Turquie, Cuba), l'actualité institutionnelle (conférences intergouvernementales, présidence de l'UE...).

Plusieurs raisons peuvent expliquer cette innovation. Tout d'abord, c'est une manière de mettre en valeur l'activité du GUE à Strasbourg (propositions, amendements, votes), et par là même de désamorcer les accusations d'euroscepticisme voire d'europhobie - nous reviendrons sur ce point. Deuxièmement, la rubrique établit systématiquement un lien entre l'activité législative européenne et les sujets nationaux (emploi, racisme, services publics...). L'un des enjeux est fonctionnel : informer les élus sur des sujets à surveiller compte tenu des nouvelles compétences européennes. Mais c'est surtout un enjeu politique : les luttes nationales voient leur signification renforcée par leur signification à l'échelon européen, tandis que les luttes européennes sont légitimées par leur impact sur le destin national. Par conséquent, les adhérents de l'ANECR sont implicitement invités à s'investir dans les débats sur l'Europe, qu'il s'agisse des élections ou des initiatives de type référendum sur l'euro en 1997-1998. C'est ainsi que dans un dossier consacré aux collectivités territoriales en 1997, L'Élu d'aujourd'hui insiste sur le rôle de l'échelon communal dans les débats sur l'Europe :

«Les élus, garants aussi de la démocratie, n'ont-ils pas à se prononcer, à donner les moyens aux populations de s'exprimer ? Le terrain des collectivités locales a été et est toujours, un territoire de grandes manœuvres pour construire une Europe lointaine, hors champ. Mais les communes sont aussi des lieux très vivaces où se défendent les opinions, en direct avec la vie de chaque jour. Les collectivités territoriales jouent un rôle central dans l'évolution permanente de l'Union européenne. $»^{13}$

La rubrique « Parlement européen » disparaît avec la nouvelle maquette adoptée en septembre 2001. Il faudrait interroger les archives et les témoins pour comprendre tous les tenants et aboutissants de cette décision. Les soucis d'économie l'expliquent probablement pour l'essentiel - la pagination est ramenée de 68 à 50 pages environ. Peut-être faut-il y voir également un changement d'époque : en janvier 2000, le mot «mondialisation» fait son apparition dans la table des matières, en surtitre à un article consacré au sommet de l'OMC à Seattle en novembre 1999. En effet, nous y reviendrons, le thème européen va progressivement s'intégrer dans la notion plus large d'anti-libéralisme.

13. « Quelle Europe ? Les collectivités territoriales, l'État et l'Europe », L'Élu d'aujourd'hui, no 212, mars 1997, p. 25. 


\section{Le PCF est-il eurosceptique?}

Le PCF a été classé dans le camp des " eurosceptiques », quand ce terme inventé par un journaliste britannique pour décrire certaines tendances nationales commença à se répandre sur le continent dans les années 1990. Par la suite, les médias forgèrent des termes analogues pour qualifier les discours critiques envers l'Europe communautaire : eurocynique, europhobique, etc., autant de discours exprimant moins un manque d'intérêt pour l'Europe qu'un doute sur l'Europe telle qu'elle est (Le Boulay, 2008 : 96).

En effet, à partir des années 1990, le processus d'intégration européenne implique un contenu explicitement politique, et plus seulement économique. Dans cette perspective, le problème du « déficit démocratique » et l'adhésion - ou non - des citoyens européens au processus deviennent un enjeu entre les partisans et les opposants à l'Europe : l'Europe sera illégitime si l'opinion publique ne la soutient pas. Ces critiques suscitent à la fois une réflexion approfondie sur l'identité européenne, et une multiplication des enquêtes sur le rapport à l'Europe.

Au-delà des slogans et des raccourcis, plusieurs lectures ont été proposées pour comprendre ces lectures de la construction européenne, notamment celle de Taggart et Szczerbiak différenciant euroscepticisme « dur » et euroscepticisme « mou » (Taggart; Szcerbiak, 2008 : 1-15) : le premier serait le rejet de toute construction européenne supranationale, le second le rejet des formes actuelles, sans remise en cause du principe de l'intégration. Dans les deux cas, l'euroscepticisme est un trait commun aux partis protestataires et un aspect de leur rejet du système en place. Cette analyse, féconde en termes d'études, a néanmoins été critiquée, en particulier parce qu'elle propose une approche transversale à tous les partis plus ou moins hostiles à l'UE, sans différencier leurs analyses et leurs approches spécifiques, et en simplifiant des attitudes qui font l'objet d'une gradation en fonction des circonstances et des époques.

Dans le cas du PCF, la typologie de Prat (Rodríguez-Aguilera de Prat, 2012: 126-127) semble plus féconde. Il invite en effet à distinguer entre l'europhobie (rejet catégorique du projet européen), l'euroscepticisme (posture critique) et l'eurocriticisme (insatisfaction sur la situation actuelle). Pour Prat, les partis politiques capturent le mécontentement et la déception des « perdants » de la construction européenne, mais d'une manière contrastée selon la tendance : les partis de droite la rejettent au nom de l'identité nationale, voire d'une forme d'ethnicité, tandis que les partis de gauche articulent leur critique autour de la notion de souveraineté. Il définit donc quatre postures : les europhiles, les europragmatiques, les eurocritiques et les europhobes. Les premiers approuvent à la fois le projet et sa mise en œuvre, les derniers rejettent l'un et l'autre. Les 
europragmatiques n'adhèrent pas au projet mais acceptent les réalisations, tandis que les eurocritiques adhérent au projet mais rejettent sa mise en œuvre actuelle.

Selon cette grille, les positions qui s'expriment dans L'Élu d'aujourd'hui oscillent entre europragmatisme et eurocriticisme tout au long de la période considérée. Francis Wurtz affirme ainsi aux journées parlementaires du PCF de septembre 1995 :

« Il est possible de créer une Europe qui soit autre chose qu'une zone de libre échange, former des projets communs, des coopérations d'intérêt mutuel. Que l'Europe puisse apporter des solutions alternatives et de poids à l'ordre mondial en développant à la fois la démocratie, la souveraineté, la solidarité. La construction de l'Europe deviendrait alors un combat moderne. ${ }^{14}$

Au printemps 1997, l'on retrouve le même appel à investir le processus européen pour le transformer de l'intérieur:

«Quels que soient les dysfonctionnements, incohérences, problèmes de fond, attaques des souverainetés nationales, l'Europe existe bel et bien. Malgré son aboutissement encore inconnu, sa construction actuelle aura été le principal événement marquant et transformant nos institutions nationales au long de la Ve République. [...] L'Europe existe. Ne s'est-t-on pas parfois désintéressé de son fonctionnement parce qu'elle est dominée par l'ultra-libéralisme ? La solution ne serait-elle pas de mettre la démocratie là où il y a actuellement diplomatie et marchandages dans les rapports avec les peuples ? ${ }^{15}$

La posture eurocritique, mais pas europhobe, reste officielle lors de la campagne contre le traité constitutionnel européen, qui articule le refus du traité à la revendication d'un nouveau texte :

«Ce « non » n'est pas un « non » à l'Europe, il est au contraire le moyen de créer les conditions de la construction d'une autre Europe, moins libérale et plus solidaire, plus efficace pour traiter des questions comme celles de l'inégalité, du développement économique et de l'emploi ou des échanges ; respectueuse des États, des cultures et des traditions de chacun des peuples européens. ${ }^{16}$

De même, Francis Wurtz, président du GUE-NGL, évoque un « besoin d'Europe » lors du Conseil national de l'ANECR en novembre 2004 :

« La construction européenne se caractérise par la dévotion au marché et l'organisation de la démission du politique. Ces deux tendances sont prolongées

14. L'Élu d'aujourd'hui, no 197, novembre 1995, p. 64.

15. « Mireille Almalan : une députée au Parlement européen », L'Élu d'aujourd'hui, no 212, mars 1997, p. 28-29.

16. Éditorial de Joseph Tréhel, « Pour une autre Europe ! », LÉlu d'aujourd'hui, no 298, janvier 2005, p. 3. 
actuellement avec le projet de Constitution européenne présenté aujourd'hui. [...] La démocratie, c'est le choix ! Or, nous avons là, outre une réforme du système institutionnel, la constitutionnalisation du modèle de l'Europe libérale. [...] Parce que je suis profondément convaincu qu'il y a, de nos jours, comme jamais, un besoin d'Europe pour espérer transformer le monde, il serait dommageable de pousser ainsi un grand nombre d'anti-libéraux à devenir anti-européens. ${ }^{17}$

Le PCF est ainsi soucieux de ne pas apparaître comme europhobe, ce qui stratégiquement le reléguerait dans la catégorie des populismes extrémistes et le couperait de son audience de centre-gauche. Jean-Claude Mairal, conseiller régional d'Auvergne et président du CIDEFE, l'exprime clairement lors du Bureau national de l'ANECR en janvier 2005 : « Le « non » peut l'emporter à condition que nous apparaissions profondément européens, en nous démarquant $\mathrm{du}$ « non » souverainiste et lepéniste ${ }^{18}$.

Pourquoi cette insistance du PCF à se dire « euro-constructif »? C'est que pour l'opinion publique et les commentateurs politiques, le concept d'euroscepticisme fonctionne comme un mot-valise permettant de jeter le même opprobre sur tous les courants politiques critiquant la construction européenne. Olivier Rozenberg l'a souligné avec ce qu'il appelle « la logique unanimiste » du vote européen : les débats de ratification des traités prennent la forme d'un vote de confiance sur la politique européenne du gouvernement. Par conséquent, en logique unanimiste, voter non, c'est soit être dans l'erreur (il faudra convaincre l'opposant qu'il se trompe pour qu'il change son vote), soit refuser le processus lui-même et les valeurs sur lesquelles il se construit ; c'est donc se mettre en marge de la communauté (Rozenberg, 2004). Par conséquent, le PCF rejette le terme d'eurosceptique, qui revient à nier le rôle qu'il entend jouer dans le débat politique. Robert l'exprimait très clairement en 1998 à propos du passage à l'euro :

«Ce n'est ni par nationalisme ni par « euroscepticisme » que le Parti communiste est opposé à l'euro. Sa critique est une contribution de gauche au débat sur l'avenir du projet européen, sa finalité sociale, ses implications pour le devenir de la nation. [...] Nous voulons restaurer un débat droite/gauche sur l'Europe. C'est la condition pour offrir aux citoyens un véritable choix. C'est à cette condition-là que nous pourrons démocratiser dans les faits la construction européenne. Je suis convaincu, a précisé Robert Hue à l'Assemblée nationale, que demain, quoi qu'il arrive, on reconnaîtra le service rendu

17. Francis Wurtz, «Constitution européenne. En matière de responsabilité citoyenne, on peut mieux faire ! », L'Élu d'aujourd'hui, no 298, janvier 2005, p. 33.

18. « Bureau national du 12 janvier 2005 : résister, élaborer », L'Élu d'aujourd'hui, no 299, février 2005, p. 44. 
à la gauche et à la France, pour n'avoir pas laissé le terrain de la critique de l'Union monétaire aux eurosceptiques et à l'extrême-droite. ${ }^{19}$

Le PCF s'efforce d'élaborer une alternative au modèle de Bruxelles. En 1994, il fait plusieurs propositions pour démocratiser les institutions : améliorer l'information et la transparence sur le processus décisionnel, limiter les pouvoirs des instances non élues (Commission, cour de justice, Banque centrale européenne) et ne pas en créer de nouvelles, étendre la possibilité du recours à l'unanimité au Conseil européen, mener les négociations intracommunautaires sous mandat des parlements nationaux ${ }^{20}$. En 1997, il propose de ne pas créer de monnaie commune mais un « instrument monétaire commun », sous forme d'un fond alimenté par les pays membres qui permettrait le financement de la recherche, des " coproductions industrielles ", le développement de régions défavorisées et la lutte contre les spéculations visant la monnaie d'un pays membre ${ }^{21}$.

L'Élu d'aujourd'hui utilise fréquemment le thème des coopérations décentralisées comme exemple de collaborations internationales exemplaires. Créées par la loi du 6 février 1992, elles permettent la signature de partenariats entre collectivités territoriales françaises et étrangères. Elles prolongent et développent la pratique des jumelages nés après la Seconde Guerre mondiale, en leur donnant un contenu plus large, en particulier en direction de l'aide au développement. Outre leur fonction propre, elles ont aussi un objectif politique explicite, actualisant les liens traditionnels du PCF avec les pays qu'il jugeait progressistes. Jean-Paul Salon, conseiller régional d'Aquitaine, le souligne lors du $12^{e}$ congrès de l'ANECR à Marseille en octobre 2003 : les coopérations décentralisées sont « le moyen de retravailler la question de l'internationalisme pour les progressistes que nous sommes en associant à la fois l'action concrète et à partir des problèmes globaux de la société $»^{22}$.

Le rapport du PCF à la construction européenne évolue donc notablement de la fin de la guerre froide au milieu des années 2000, sous l'effet de plusieurs facteurs : sa transformation interne, son expérience propre des institutions européennes, la transformation du contexte international et le rôle désormais plus discret de l'idéologie. Mais les questions européennes ne sont pas

19. Transparence, no 3, mai-juin 1998, p. 2.

20. « Une communauté de nations souveraines et partenaires. Entretien avec Francis Wurtz, député européen », L'Élu d'aujourd'hui, no 181, mai 1994, p. 27.

21. L'Élu d'aujourd'hui, no 215, juin 1997, p. 40.

22. « Construire des points d'appui pour un monde meilleur », L'Élu d'aujourd'hui, no 285, novembre 2003, p. 30. 
seulement un thème politique : au fil des années, l'Europe devient un enjeu à la fois interne et externe.

\section{L'Union européenne comme thème stratégique}

Les élections européennes jouent un rôle stratégique important car elles sont à la fois un moment de visibilité et une opportunité financière pour les petits partis. Lusage de la proportionnelle pour les élections au Parlement européen, ainsi que le soutien financier public pour les campagnes, favorisent des campagnes actives et garantissent une couverture médiatique propices à la diffusion de leurs positions, surtout dans un contexte de controverse (Reungoat, 2019). De plus, le statut d'élu européen procure un certain nombre de ressources matérielles, politiques et symboliques, dont on a vu que le PCF avait su faire usage.

Sous un angle plus large, les élections européennes peuvent être l'occasion de se renforcer en tant que parti d'opposition. En effet, dans la mesure où elles ne modifient pas l'équilibre des forces politiques au pouvoir, les élections européennes sont souvent l'occasion d'un vote-sanction envers le gouvernement, indépendamment des enjeux européens proprement dits. S'abstenir, voter blanc ou voter pour des partis d'opposition sont autant de manières de contrer la politique officielle et la «pensée unique » pro-européenne commune aux grands partis, sans réels risques en termes de politique nationale.

Le plus grand risque, pour le PCF, est que les électeurs choisissent l'abstention ou le vote blanc. Les campagnes électorales sur l'Europe doivent convaincre que le vote communiste est un vote à la fois protestataire et utile : «Voter le 12 juin pour la liste présentée par le PCF c'est la façon la plus sûre d'affirmer son opposition à la politique de monsieur Balladur et de faire grandir une composante communiste forte et active au sein des forces de gauche $»^{23}$, explique L'Élu d'Aujourd'hui pour les européennes de 1994. Larticulation entre vote européen et vote national n'est cependant pas toujours aisée, témoin cet éditorial en vue des européennes de 2004 :

« Il nous faut... travailler à agir et à rassembler pour un vote de construction d'une alternative progressiste qui sera en même temps un vote de résistance à la politique actuelle et point d'appui pour les luttes à venir afin de faire reculer le pouvoir tout de suite. $»^{24}$

LEurope est un thème stratégique pour une deuxième raison : elle contribue à structurer les débats, notamment sur la question des alliances politiques.

23. «Vie de l'ANECR. De la vie quotidienne au vote pour la liste conduite par Francis Wurtz », L'Élu d'aujourd'hui, no 181, mai 1994, p. 10.

24. « Agir et rassembler », L'Élu d'aujourd'hui, no 287, janvier 2004, p. 3. 
Nicolas Azam a minutieusement mis en valeur la manière dont le thème européen est un enjeu politique interne au PCF, dès les années 1980 et a fortiori après 1991 : l'Europe est à l'époque une ligne de partage entre refondateurs et « économistes» autour de Philippe Herzog (Azam, 2017 : 492). Le virage vers un soutien critique à l'UE, à partir de 1995-1996, ne va pas non plus sans remous, et les oppositions internes s'en emparent pour argumenter en faveur d'un « retour aux fondamentaux» du PCF (Azam, 2017 : 578). La vaste étude menée par François Platone et Jean Ranger sur les militants PCF en 1997 atteste de ces clivages concernant l'idée du parti : une minorité importante des militants (20\%) est hostile aux transformations récentes, restant attachée notamment à la notion de rôle dirigeant de la classe ouvrière, et estime à $90 \%$ que l'appartenance à l'Europe n'a pas bénéficié à la France, contre 70 \% chez les adhérents récents (Platone ; Ranger, 2000).

L'un des principaux débats est celui des alliances stratégiques : faut-il privilégier l'alliance avec le Parti socialiste, donc une attitude ouverte sur la construction européenne? Ou bien faut-il rechercher la formation d'un front d'extrême-gauche, mais dans ce cas durcir le discours ?

La participation à la majorité de la « gauche plurielle » entre 1997 et 2002 a permis au PCF de revenir au pouvoir avec trois ministres communistes dans le gouvernement de Lionel Jospin. Mais la solidarité gouvernementale le conduit à soutenir des politiques contraires aux vœux de son électorat. C'est ainsi que les députés du PCF s'abstiennent lors du vote sur le traité de Nice en 2001, au lieu de voter comme le font de nombreux partis communistes européens (Reungoat, 2019 : 78).

La même situation s'observe autour de la chasse. C'est un sujet sensible pour des élus ruraux, qui revient à plusieurs reprises dans L'Élu d'aujourd'hui. Le PCF a toujours combattu la directive européenne de 1979 protégeant les oiseaux migrateurs, au nom de la défense des traditions nationales ${ }^{25}$. De plus, la chasse permet d'illustrer la distance entre la technocratie bruxelloise déshumanisée et les citoyens connaisseurs du terrain. Un communiqué du GUE en 1996 l'illustre bien, qui refuse une réglementation européenne commune pour un sujet dont les modalités locales varient ${ }^{26}$.

Quatre ans plus tard, le PCF est toujours sur cette ligne, qui permet d'insérer la chasse dans le cadre plus large de l'analyse de classe. Ce qui menace avant tout la biodiversité, explique Jean Querbes, ancien député européen, c'est la bétonisation portée par des « partisans de la rentabilité et de la productivité

25. « Le droit aux chasses traditionnelles », LÉlu d'aujourd'hui, no 139, juillet-août 1990, p. 9. 26. Mireille Elmalan, "La chasse doit tenir compte des spécificités locales », L'Élu d'aujourd'hui, no 201, mars 1996, p. 65. 
à tout prix $»^{27}$. Les interdictions croissantes issues de la directive 79-409 cherchent à diminuer la place d'une « chasse populaire et démocratique » et plus largement le « contrat social sur l'accès à l'espace naturel », pour aller vers « une chasse privée sur des territoires fermés et seulement accessibles à ceux qui en ont les moyens financiers » : on reviendrait au système d'Ancien Régime, les «privilèges de la noblesse » étant remplacés par « les privilèges de l'argent $»$.

Ces prises de position contribuent à l'ancrage en milieu rural, comme Julian Mischi et Julian Weisbein l'ont montré à travers le cas du vote communiste en marais de Brière. Mais lorsqu'en 2000 le PCF s'abstient sur la loi chasse qui réduit les dates d'ouverture, afin de ne pas se dissocier de la majorité de la " gauche plurielle », il perd immédiatement ses appuis locaux au profit de Chasse, Pêche, Nature et Tradition (Mischi ; Weisbein, 2004).

De fait, les européennes de 1999 sont un échec et une déception. La liste "Bouge l'Europe ", doublement paritaire (hommes et femmes, membres du parti et membres extérieurs) était censée mettre en valeur la « mutation » du PCF, et une forme de dépassement de Maastricht : de manière symbolique, la philosophe Geneviève Fraisse y était deuxième sur la liste, alors qu'elle avait voté oui en 1992. Mais la liste communiste n'attire pas plus de suffrages qu'en 1994. Officiellement, Robert Hue l'explique par l'abstention de l'électorat de gauche sceptique sur la possibilité d'une Europe sociale, et le report d'une partie des voix sur les Verts à cause des récents scandales alimentaires (vache folle $)^{28}$. Pire, les élections voient une progression inédite de l'extrême-gauche qui rassemble plus de $5 \%$ des suffrages, soit 5 députés.

En 2003, l'échec cuisant de Robert Hue aux présidentielles amène MarieGeorge Buffet à la présidence du Bureau national et, avec elle, une ligne plus critique. L'effervescence des alternatives altermondialistes et les mobilisations communes conduisent progressivement le PCF à inverser son alliance, de sa droite vers sa gauche, du PS vers la nébuleuse altermondialiste.

Né sur le terreau de la gauche, l'antilibéralisme a des filiations multiples (marxisme, tiers-mondisme, dénonciations du capitalisme et du libéralisme économique), mais son ciment commun est le rejet des politiques et des institutions encourageant la dérégulation des échanges. Lintégration européenne favorise l'ouverture intérieure entre pays membres, tout en entretenant des barrières protectrices vis-à-vis de l'extérieur (par exemple tarif extérieur commun,

27. Jean Querbes, « Faux débat sur la chasse et vrai débat de société », L'Élu d'aujourd'hui, no 244, février 2000, p. 17.

28. Robert Hue, «Les élections européennes de 1999 ", L'Élu d'aujourd'hui, no 238, juillet-août 1999, p. 60. 
subventions agricoles, normes techniques). Mais par définition, les « compétences exclusives » de l'UE dépossèdent les États membres de certains leviers d'action, par exemple les droits de douane, pour protéger leur marché. Les traités successifs, Maastricht, Amsterdam, Nice, élargissent progressivement le domaine des compétences exclusives de la Commission européenne, tout en rendant plus complexe le processus décisionnel et en suscitant une inflation de textes et de réglementations juridiques et techniques de lecture ardue.

Pour ceux qu'elle fragilise, la mondialisation est une forme de dépossession : les citoyens sont menacés par des phénomènes se produisant hors des frontières nationales et qui, par le jeu des intrications diverses, les affectent directement et entretiennent la crise sociale. C'est précisément dans ce contexte que se développe un foisonnement d'associations qui, à travers des activismes variés, cherchent à proposer des solutions à la précarité et des alternatives au modèle néo-libéral. Elles constituent le socle d'ATTAC (Association pour la taxation des transactions financières et pour l'action citoyenne) créée en France en 1998. Lantilibéralisme est le point fédérateur de cette nébuleuse où se rencontrent à la fois les colères du terrain et l'expertise économico-politique (Mathieu, 2005 : 153).

$\mathrm{Au}$ Conseil européen de Bruxelles le 18 juin 2004, les chefs d'État et de gouvernement de l'UE adoptent le texte du Traité établissant une constitution pour l'Europe ; il sera formellement signé le 29 octobre. Dès le printemps, il est clair que le PCF rejettera le texte :

« Le projet de Constitution reprend tout simplement le texte du traité de Maastricht : les politiques partent des exigences des marchés et le fonctionnement se fait par le haut. [...] Pire, il y a « constitutionnalisation» du modèle libéral. $»^{29}$

Lors des journées parlementaires des 28 et 29 septembre 2004 réunissant députés et sénateurs communistes, les divergences internes sur la construction européenne se révèlent :

"Dénonçant une certaine cacophonie au sein du parti, le député-maire de Saint-Denis, Patrick Braouzec, a reconnu que « sur certaines questions contemporaines, les parlementaires communistes ne partagent plus les mêmes valeurs ». Réclamant une " clarification ", il a souhaité que soit demandé aux communistes s'ils sont " pour ou contre une autre constitution européenne ", manière de faire la distinction entre les opposants au traité signé à Bruxelles et les anti-européens purs et simples. $»^{30}$

29. Francis Wurtz, «L'Europe appartient à ses habitants », L'Élu d'aujourd'hui, no 289, mars 2004 , p. 33.

30. « Être plus offensif et plus lisible », LÉlu d'aujourd'hui, no 295, octobre 2004, p. 39. 
Plus révélatrice encore, la différence d'analyse entre Francis Wurtz et MarieGeorges Buffet. Le député européen appelle à " rassembler toute la gauche dans l'opposition à cette constitution européenne », et les réserves de Laurent Fabius sur le TCE «montre[nt] que le non peut l'emporter». Au contraire, la secrétaire nationale du PCF pose explicitement les jalons d'une réorientation vers l'extrême-gauche :

« "Il faut travailler dès maintenant à construire un rassemblement populaire majoritaire porteur d'une politique de transformation sociale ", note MarieGeorge Buffet qui accuse, sans le nommer, le PS de vouloir " entraîner toute la gauche vers une politique social-libérale. " $»^{31}$

La campagne contre le TCE est lancée à l'automne 2004 : le 19 octobre 2004, un Comité national pour un «non » de gauche, sous l'égide de la fondation Copernic, lance un « appel des $200 »$. Les signataires sont représentatifs de la mouvance anti-libérale, à la dynamique récente et multiforme (partis, associations, fondations, collectifs...). Dès novembre 2004, L'Élu d'aujourd'hui fait sa couverture sur ce sujet, avec un dossier intitulé "Construire un rassemblement antilibéral ». Le Bureau national de janvier 2005 fait du non l'une de ses « batailles » de l'année à venir, dans le cadre d'une stratégie plus large de réorientation politique : « œuvrer à l'émergence d'une alternative politique ; résister et s'opposer aux visées du gouvernement et du Medef ; élaborer des propositions porteuses d'avancées politiques immédiates et de changement plus profond $»^{32}$.

Pour le PCF, c'est un choix stratégique, qui ne va pas sans dissensions internes : les partis d'extrême-gauche, notamment la LCR, sont bien implantés dans cette mouvance, ce que les plus orthodoxes désapprouvent. D'autre part, il faut conserver un discours partisan face à ATTAC qui se revendique comme apolitique. Mais l'expérience commune et les réseaux locaux tissés dans les luttes sociales contribuent à encourager le courant réformateur favorable à cette ouverture (Reungoat, 2019 : 133). Au lendemain de la victoire du non le 29 mai 2005, l'on peut rêver d'une candidature unitaire d'extrême-gauche aux présidentielles de 2007 : «L'organisation de la riposte va de pair avec celle de la construction d'un mouvement progressiste prenant appui sur une base résolument anti-libérale. $»^{33}$

31. Ibid.

32. « Bureau national du 12 janvier 2005 : résister, élaborer », L'Élu d'aujourd'hui, no 299, février 2005, p. 44.

33. Éditorial de Joseph Tréhel, « Amplifions le mouvement ! », L'Élu d'aujourd'hui, no 304, juillet 2005, p. 3. 


\section{Conclusion}

Retracer l'histoire de la perception de l'intégration européenne par le PCF permet ainsi de requestionner plusieurs affirmations classiques : c'est un parti eurosceptique ; il est resté le gardien d'une orthodoxie communiste sans modifier structurellement ses analyses ; l'Europe est un sujet stratégique secondaire, puisque les élections européennes attirent moins que les élections nationales.

$\mathrm{Au}$ contraire, le discours critique du PCF sur l'Europe évolue profondément, passant du souverainisme à l'anti-libéralisme, de l'analyse géopolitique à la défense des droits sociaux. Le thème européen est au cour des controverses intrapartisanes, mais aussi l'objet de paris électoraux : mettre en scène la modernisation en 1999, rassembler le non de gauche en 2004-2005. Il joue également un rôle clé dans l'évolution stratégique du parti : en 2005, ce sont les électeurs de gauche, notamment ceux du Parti socialiste, qui ont été décisifs dans la victoire du non (Perraudeau, 2006). Tandis qu'en 1992 le PCF votait non avec les partis de droite, en 2005 il s'intègre dans une dynamique commune à toutes les tendances de la gauche.

On peine cependant à discerner le projet du PCF concernant l'Europe, et quelles politiques pourraient concrétiser, à l'échelle des institutions, les mots d'ordre qui séduisent son électorat décroissant. D'autre part, le prisme d'une revue précise peut conduire à surestimer l'importance relative de certains thèmes. L'Élu d'aujourd'hui a clairement été une tribune pour la tendance la plus « européiste » du PCF, mais il faudrait analyser plus en profondeur la place de la revue dans les jeux de pouvoirs et d'influences internes au parti, et la réception des idées exposées. L'on ne peut que souhaiter davantage de recherches sur la dimension vécue de l'action politique. 
Fin de l'histoire et nouveaux temps : le PCF et l'intégration européenne du traité...

Annexe : Évolution du vote communiste de 1978 à 2009

\begin{tabular}{|c|c|c|c|c|}
\hline Date & Élections & $\%$ des exprimés & Nombre de sièges & Nombre d'adhérents \\
\hline 1978 & Législatives & $20,60 \%$ & 86 & 566492 \\
\hline 1979 & Européennes & $20,50 \%$ & 19 & \\
\hline 1981 & Présidentielles & $15,35 \%$ & & \\
\hline 1981 & Législatives & $16,10 \%$ & 44 & \\
\hline 1982 & & & & 457864 \\
\hline 1984 & Européennes & $11,20 \%$ & 10 & \\
\hline 1986 & Législatives & $9,80 \%$ & 35 & \\
\hline 1988 & Présidentielles & $6,76 \%$ & & \\
\hline 1988 & Législatives & $11,30 \%$ & 25 & \\
\hline 1989 & Européennes & $7,70 \%$ & 7 & 369167 \\
\hline 1993 & Législatives & $9,20 \%$ & 25 & \\
\hline 1994 & Européennes & $6,90 \%$ & 7 & 281004 \\
\hline 1995 & Présidentielles & $8,640 \%$ & & \\
\hline 1997 & Législatives & $9,90 \%$ & 35 & 225394 \\
\hline 1999 & Européennes & $6,80 \%$ & 6 & \\
\hline 2001 & & & & 147511 \\
\hline 2002 & Présidentielles & $3,37 \%$ & & \\
\hline 2002 & Législatives & $4,80 \%$ & 21 & \\
\hline 2004 & Européennes & $5,40 \%$ & $2(a)$ & \\
\hline 2007 & Présidentielles & $1,92 \%$ & & \\
\hline 2007 & Législatives & $4,30 \%$ & 15 & \\
\hline 2009 & Européennes & $6,00 \%$ & $4(b)$ & 133476 \\
\hline
\end{tabular}

Sources : le nombre d'adhérents est issu des travaux synthétisés dans Martelli ; Vigreux ; Wolikow, 2020, à partir d'une enquête minutieuse des auteurs dans les archives du PCF. Les résultats électoraux sont issus des données officielles proposées en ligne par le Parlement européen (https://www.europarl.europa.eu/about-parliament/fr/in-the-past/ previous-elections).

(a) 3 sièges avec l'appoint de l'élu Union de la gauche-Parti communiste réunionnais.

(b) Liste commune "Front de gauche pour changer d'Europe » 


\section{Références}

AZAM, Nicolas (2017). Le PCF confronté à l' "Europe ». Une étude socio-historique des prises de position et des recompositions partisanes, Paris : Dalloz.

BEAUVALLET, Willy; MICHON, Sébastien (2013). Les usages politiques de l'Europe par les eurodéputés de la gauche eurocritique. L'exemple des élus Français de la Gauche unitaire européenne entre 1979 et 2004. In Maria GAINAR, Martial LIBÉRA (Eds.). Contre l'Europe ? Anti-européisme, euroscepticisme et alter-européisme dans la construction européenne, de 1945 à nos jours, vol. 2 Acteurs institutionnels, milieux politiques et société civile (127-139). Stuttgart : Franz Steiner Verlag. https://halshs.archives-ouvertes.fr/halshs-00856343

COOSEMANS, Thierry (2004). Lélection du Parlement européen de juin 2004. Courrier hebdomadaire du CRISP, 18 (1-66). https://doi.org/10.3917/ cris. 1843.0005

LAVABRE, Marie-Claire; PLATONE, François (2003). Que reste-t-il du PCF ?. Paris : Autrement.

LAZAR, Marc (2000). La galaxie du communisme et de l'extrême-gauche. In Gérard GRUNBERG; Pascal PERRINEAU ; Colette YSMAL (Eds.). Le vote des Quinze. Les élections européennes du 13 juin 1999 (121-134). Paris : Presses de Sciences Po.

LE BOULAY, Morgane (2008). La fabrication d'un label. Usages du terme « euroscepticisme » en France et en Allemagne. In Laure NEUMAYER, Antoine ROGER, Frédéric ZALEWSKI (Eds.). LEurope contestée. Espaces et enjeux des positionnements contre l'intégration européenne (87-113). Paris : Michel Houdiard.

MARTELLI, Roger ; VIGREUX, Jean; WOLIKOW, Serge (2020). Le Parti rouge. Une histoire du PCF, 1920-2020. Paris : Armand Colin.

MATHIEU, Lilian (2005). La constitution du mouvement altermondialiste français. Critique internationale, 27, 147-161. https://doi.org/10.3917/crii.027.0147

MISCHI, Julian ; WEISBEIN, Julien (2004). LEurope comme cause politique proche : contestation et promotion de l'intégration communautaire dans l'espace local. Politique européenne, 12 (84-104). https://doi.org/10.3917/ poeu.012.0084

MISCHI, Julian (2007). Pour une histoire sociale du déclin du parti communiste. In Florence HAEGEL (Eds.). Partis politiques et système partisan en France (69-101). Paris : Presses de Sciences Po.

PERRAUDEAU, Éric (2006). Les Français dans le miroir européen. Le référendum du 29 mai 2005. Pouvoirs, 116 (149-161). https://doi.org/10.3917/ pouv.116.0149

PLATONE, François ; RANGER, Jean (2000). Les adhérents du Parti communiste français en 1997. Paris : Sciences Po Paris. 
RODRÍGUEZ-AGUILERA DE PRAT, Cesáreo (2012). Euro-escepticismo, Eurofobia y Eurocriticismo. Los partidos radicales de la derecha y la izquierda ante la Unión Europea. Barcelona : Huygens Editorial.

PUDAL, Bernard (2009). Un monde défait. Les communistes français de 1956 à nos jours. Vulaines-sur-Seine : éditions du Croquant.

REUNGOAT, Emmanuelle (2019). Enquête sur les opposants à l'Europe. À droite et à gauche, leur impact d'hier à aujourd'hui. Lormont: Le Bord de l'Eau.

ROBIN, Émilia (2009). Anti-européens et euroconstructifs : les communistes français et l'Europe (1945-1979). Cahiers Irice, 4 (49-67). https://doi.org/10.3917/ lci.004.0049

ROZENBERG, Olivier (2004). « Du non-usage de l'Europe par les parlementaires nationaux : la ratification des traités européens à l'Assemblée nationale ». In Sophie JACQUOT ; Cornelia WOLL (Eds.). Les usages de l'Europe. Acteurs et transformations européennes (261-292). Paris : L'Harmattan.

ROZENBERG, Olivier (2007). La faute à Rousseau ? Les conditions d'activation de quatre idéologies critiques de la construction européenne en France. In Justine LACROIX ; Ramona COMAN (Eds.). Les résistances à l'Europe. Cultures nationales, idéologies et stratégies d'acteurs (129-153). Bruxelles : éditions de l'université de Bruxelles.

TAGGART, Paul ; SZCZERBIAK, Aleks (2008). Opposing Europe? The Comparative Party Politics of Euroscepticism. Oxford : Oxford University Press. 